\title{
Mast cells positive to Tryptase correlates with protease-activated receptor-2 expression and microvascular density in breast cancer patients
}

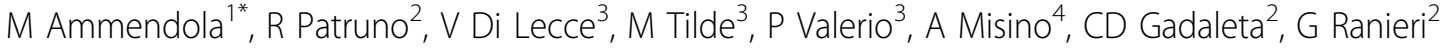 \\ From de Senectute: Age and Health Forum \\ Catanzaro, Italy. 5-7 December 2009
}

\begin{abstract}
Background
Angiogenesis is an important pathway in tumour growth and progression. Angiogenesis is regulated by several classical factors, Vascular Endothelial Growth Factor being the most important. On the other hand tryptase, a serine protease stored and released from mast cells (MCs) granules has been identified as a new non-classical angiogenetic factor. Tryptase is an agonist of the proteinase-activated receptor-2 (PAR-2) a $\mathrm{G}$ protein involved in cellular proliferation and angiogenesis. In this study, we have evaluated the correlations between the number of MCs positive to tryptase (MCDPT), the number of breast cancer cells positive to PAR-2 (BC-PAR-2) and microvascular density (MVD) in a series of 97 primary T1-3, N0-2 M0 female breast cancer by means of immunohistochemistry and image analysis methods.
\end{abstract}

\section{Materials and methods}

Six-micrometers thick serial sections of formalin-fixed and paraffin-embedded bioptic tumor samples were obtained. Then sections were microwaved at $500 \mathrm{~W}$ for $10 \mathrm{~min}$. and treated with a $3 \%$ hydrogen peroxide solution. Sections were incubated with primary human-specific antibodies: monoclonal anti-tryptase (clone AA1; Dako, Glostrup, Denmark), goat polyclonal anti NH2 terminal of PAR-2 (clone N-19; sc-8206 Santa Cruz Biotecnology), and monoclonal anti-CD34 (QB-END 10; Bio-Optica Milan, Italy). Biotinylated secondary antibody, avidin-biotin peroxidase complex, and 3-amino-9-ethylcarbazole were in turn utilised. In serial sections "hot spots" were selected at low magnification and both individual vessels, single tryptasepositive MCs and breast cancer cells positive to PAR-2 were counted at $\times 400$.

${ }^{1}$ Clinical Pharmacology Unit, University Magna Graecia of Catanzaro Medical School, Catanzaro

Full list of author information is available at the end of the article

\section{Results}

Data demonstrated a significant correlation between MCDPT, BC-PAR-2 and MVD to each other $(r=$ ranging from 0.71 to 0.87 ; p: ranging from 0.001 to 0.003 by Pearson's analysis respectively). No correlation concerning MCDPT, BC-PAR-2, MVD and the main clinical pathological features was found.

\section{Conclusions}

Published in vitro data suggest that tryptase may increase capillary growth and endothelial cell proliferation. On the other hand tryptase induce angiogenesis by activation of PAR-2 in vascular endothelial cells and breast cancer cells lines. According to these experimental data our results suggest that tryptase-positive MCs, PAR-2 and MVD paralleled to each other thus underlying a role in in vivo breast cancer angiogenesis. In this context several tryptase inhibitors such as gabexate mesilate and nafamostat mesilate might be evaluated in clinical trials as a new antiangiogenetic drugs.

\section{Acknowledgements}

This work was supported in part by grants of Alleanza Contro il Cancro Istituto Superiore di Sanità, Ministero della Salute, Italy.

\section{Author details}

${ }^{1}$ Clinical Pharmacology Unit, University Magna Graecia of Catanzaro Medical School, Catanzaro. ${ }^{2}$ Interventional Radiology Unit with Integrated Section of Medical Oncology, National Cancer Institute Giovanni Paolo II, Bari, Italy.

${ }^{3}$ Surgey Unit, Di Venere Hospital, Bari Italy. ${ }^{4}$ Oncology Unit, National Cancer Institute Giovanni Paolo II, Bari, Italy.

Published: 27 August 2010

doi:10.1186/1471-2318-10-S1-A114

Cite this article as: Ammendola et al: Mast cells positive to Tryptase correlates with protease-activated receptor-2 expression and microvascular density in breast cancer patients. BMC Geriatrics 2010 10(Suppl 1):A114. 